\title{
Reflexive-Integrative Approach to the future IT-specialists teaching
}

\author{
Irina Kibal'chenko*, Tatiana Eksakusto \\ Southern Federal University, Institute of computer technologies and information security, \\ 347922 Taganrog, Russia
}

\begin{abstract}
The article is aimed at substantiating the idea of reasonability of the reflexive-integrative approach using in teaching the future IT-specialists. This approach is studied as the students' experience forms integration reflected in conceptual thinking, reflexion, reflexive value system, conceptualization style actualization in a process of studying. The research is focused on studying the empirical results of using the reflexive-integrative approach on the basis of students drawing up the ampliative texts including key words of conceptual field, characterizing learning process. Two groups of students (studying information systems and technologies) took part in the research: $1^{\text {st }}$ group control group (50 persons); $2^{\text {nd }}$ group - experimental one (50 persons). It is found out that the experimental group is characterized by a significant reflexivity change (increase in indices) as well as by conceptualization variation in a course of developing and categorizing the list of words, characterizing the "education" notion conceptual field. The article proves that the reflexiveintegrative approach using in a learning process improves and enlarges informative capacity of training classes as well as helps to check individuality and uniqueness of each learning process, subject's experience structure and concept content.
\end{abstract}

Today's social and economic changes lead to the following necessary requirements: society needs expansion in the number of highly-professional specialists and professionals able to solve creative and analytic cognitive tasks of high complexity. Taking into account the development of digital economics, the requirement mentioned above is of great importance and is to be payed attention to in a process of teaching the future IT-specialists [1]. The development of students' self-sustainability, creativity, ability to make out-of-the-box solutions involved rejection of the outdated forms of teaching, improving the educational system, including innovative technologies and methods in the process of education with the aim to develop students' intellectual and personal resources [2, 3]. Contemporary educational standards fortify the necessity of developing the students' universal metasubject competences including integrated subsystems of hard and soft skills. One of the basic approaches meeting the modern requirements of digital economics and educational standards in teaching IT-specialists is the reflexive-integrative approach focused on reflection and conceptual thinking studied as the integration foundations defined by several common bases [4].

\footnotetext{
* Corresponding author: kibal-irina@mail.ru
} 
The matter point of the reflexive-integrative approach is the necessity to create supportive and comfortable conditions of educational activity to involve students into mental activity, sensual and emotional processes, supposing students mastering the problem of personal experience "isolation", "stratification" and "conflict" on the conceptual, introspective and reflective levels. The approach studied in the research is aimed at establishing conditions for different experience forms integration by actualizing conceptual thinking, reflection, reflective co-operation, reflective value system, etc., in the course of a learning process. Having analyzed the conceptual thinking and the reflexive value from the point of view of personal experience (including different forms of it), content of education and conceptual field (important external resource), the student is able to create an image of current circumstances; to understand and to make a model of these circumstances in a system of the overall learning process.

Reflexive-integrative approach makes the actualization of scientifically grounded and predictive mechanisms possible, it helps teachers and students to study themselves and their experience intensively with the aim to understand, perceive this experience, analyze it incisively, reflect and evaluate. Reflexion and integration, integration and differentiation are correlative, they are closely connected and provide a basis for students' cognitive structures (understood as structural integrity or "subsystems" if using systems approach language) progressive development; these cognitive structures are new and of higher level [5].

Using the reflexive-integrative approach is efficient and topical for teaching the future ITspecialists with well-developed separate mental resource elements or components, e.g., conceptual, metacognitive or intentional ones [6]. The authors use the reflexive-integrative approach in the future IT-specialists teaching process as the way to develop their conceptual abilities (by means of improving reflexivity and conceptualization style); this approach is focused on students subjective processing of class information and assignments. According to this approach, each student is characterized by specific and peculiar conceptual experience as well as by personal and unique ability to process and learn education material. Students get the following project task: to formulate the set of words characterizing the field of particular notion (the notions can be different, e.g., "education", "technologies", "system", "information", etc.) and to find out categorization of these words. The students are aimed at consolidating the educational information and coming to categoric and conceptual levels of understanding the discipline "Psychology of personal resources managing". The Free Sorting Test (Gardner, Holzman, Klein, Linton, Spence) aimed at assessing consistent preferences for broad versus narrow equivalence ranges as well as a variation of this test (by V.A. Kolga) were used as a theoretic basis for this research [7].

The following hypothesis was put forward during our research: the reflexive-integrative approach implementation through students drawing up the ampliative texts with the key words from conceptual field referential to teaching and learning processes is the key condition for the future IT-specialists' reflexivity and conceptualization style being developed as the signs of conceptual abilities.

One hundred students of the Southern Federal University took part in the research; these students were divided into two groups: control group ( $n=50$ persons) and experimental one ( $n=50$ persons). The following methods and techniques were used:

- the method of parallel groups and random assignment was used for preliminary qualification of undistinguishable (almost homogeneous) variables;

- statistical methods (Wilcoxon's T-criterion was used to define reliable positive or negative changes in the results before and after using technological approach; Fisher (angular) transformation criterion $\left(\varphi^{*}\right.$ criterion) was aimed at finding out significant difference in the results);

- V.A. Kolga's variation of the Free Sorting Test using was aimed at conceptualization style qualifying as a form of compulsory intellectual control (analytic/synthetic characters) [8]; 
- A.V. Karpov's questionnaire to distinguish reflexivity [9].

While analyzing the reference data, the attention was to be payed to the following fact: the results of distinguishing conceptualization style (0.32 and 0.34 ) and reflexivity (4.32 and 4.34) at the first step of the research (before using reflexive-integrated approach in a learning process) were almost equal in both groups, characterized by predominance of synthetic conceptualization style. The words were sorted formally or topically, this fact shows that the estimation criteria prevail in this case. Reflexivity indices are on the average level in both groups, i.e., the students are able to fulfil reflexive tasks, analyze the current situation and estimate personal attitude to this situation but they do it incorrectly.

Technological way of the reflexive-integrative approach implementation was used at the second step of the research and was applied to the experimental group. The integrative notion of "education" was used as the key one; this notion included humanitarian and technical knowledge (the students are able to show their competence in general studies as well as in restricted professional ones dealing with this notion). This way of the reflexive-integrative approach implementation was not applied to the participants of the second (control) group characterized by the same launch environment.

The experimental group was divided into subgroups (the students in these subgroups swapped over with the aim to take on each of the following roles): diagnostic text developers (1 to 9 persons), experts (to apprise the developed texts -7 to 9 persons); respondents (to approve the texts practically -42 persons).

The students under test were given an instruction: they had to take into account the fact that there is no silver bullet. Each student was able to define semantic field of the key word and subdivide this semantic field into the word groups from the point of view of their own learning and cognitive experience.

The subgroup of developers fulfilled the following functions: chose the correct words to characterize the key word and define the field of the notion expressed by this key word; gave the list of these words to the experts to be apprised; after that they categorized the words chosen from their list and apprised by the experts; described the key to process the results.

For example, students dealt with developing the set of words describing the "education" concept. The number of developers: 1 person.

39 words were suggested at the first step: academy, library, upbringing, proficiency, dean, diploma, discipline, availability, knowledge, learning, institution, intelligence, research, quality, qualification, concept, culture, science, teaching, liability, experience, report, subject, reputation, progress, program, planning, development, term exams, student, level, system, rationalization, article, theory, improvement, teacher, lesson, school.

The subgroup of experts ( 7 persons) made the list of words more accurate: they added new words (total 15: transcript, elementary (education), humanitarian (education), technical (education), economic (education), thesis work, Bachelor, Biology, exam, erudition, tests, Physics, specialist, Chemistry, Master); and stripped off two words from the original list (studying, report).

Each step of the research included student's reflexion and conceptual abilities updating while fulfilling the tasks.

Having analyzed and compared the results of each step, we got the total quantity of words 52 (i.e., 100 per cent). There were 39 words at the first step ( 75 per cent); 15 words were added to and 2 words were excluded from the list, i.e., total rate of changes is 25 per cent.

Significant difference in the results of the first and the second steps of the research was obtained as owing to Fisher (angular) transformation criterion applying: significantly small quantity of words was excluded:

Significantly fewer words were added to the list:

$$
\varphi^{*}{ }_{\mathrm{emp}}=3.205, p \leq 0.01
$$

$$
\varphi^{*}{ }_{\mathrm{emp}}=4.78, p \leq 0.01
$$


The developer offered 11 groups and named them at the third step of the research; experts specified these groups: added 4 new groups and edited 3 ones.

As a result, the following list of groups was made:

1. Functions of education: upbringing, teaching.

2. Checking: term exams, tests, exam and erudition.

3. Information: lesson, experience, theory, knowledge, research.

4. Innovation: science, progress, development, improvement.

5. Role: teacher, dean, student.

6. Structure: system, level, qualification.

7. Institution: school, institute, academy, library.

8. Requirements: availability, quality, liability.

9. Standard: planning, program, concept.

10. Status: reputation, proficiency, intelligence, culture.

11. Studying: discipline, rationalization.

12. Scope of education: elementary, economic, technical, humanitarian.

13. Level: specialist, Bachelor, Master.

14. Content of education: Chemistry, Biology, Physics, subject.

15. Report (result): diploma, article, transcript, thesis work.

Significant difference at the third step of the research was obtained as owing to Fisher (angular) transformation criterion applying: significantly small number of groups was improved by experts:

as well as significantly few groups were added to the list:

$$
\varphi^{*}{ }_{\text {emp }}=2.209, p \leq 0.05
$$

$$
\varphi^{*}{ }_{\text {emp }}=2.656, p \leq 0.01
$$

The stimulus material was given to a group of students (42 persons) at the final step of the research to be practically evaluated (the words were offered to be grouped as the students may in their discretion think fit).

Two types of groups were defined after analyzing the results: 15 groups congruent in names with the author and experts' opinion; 14 groups specifying and broadening the semantic field of the "education" notion (educational establishment, learning process, steps of getting education, knowledge evaluation, natural sciences, level of education, assignment, documents, control, development, etc.).

Significant difference between the results of categorization coincide with the "key" and groups specifying the semantic field of the "education" notion at the evaluation step was obtained according to Fisher (angular) transformation criterion:

$$
\varphi^{*}{ }_{\text {emp }}=3.166, p \leq 0.01
$$

These results reflect gradual changes in students' conceptualization styles in a course of training events.

Additional assessment of reflexivity and conceptualization styles was held to prove the suggested hypothesis. The results are shown in Table 1.

Table 1. The results of the first and the second assessments in two groups

\begin{tabular}{|l|l|l|l|l|}
\hline Groups & Variables & $\begin{array}{l}\text { Results before the } \\
\text { project task fulfilling }\end{array}$ & $\begin{array}{l}\text { Results after the } \\
\text { project } \\
\text { fulfilling }\end{array}$ & Changes \\
task & & \\
\hline $\begin{array}{l}1 \\
(50 \text { persons })\end{array}$ & $\begin{array}{l}\text { Conceptualization } \\
\text { style }\end{array}$ & 0.32 & 0.78 & increasing \\
\cline { 2 - 5 } & Reflexivity & 4.32 & 5.34 & increasing \\
\hline $\begin{array}{l}2 \\
(50 \text { persons })\end{array}$ & $\begin{array}{l}\text { Conceptualization } \\
\text { style }\end{array}$ & 0.34 & 0.42 & $\begin{array}{l}\text { not } \\
\text { significant }\end{array}$ \\
\cline { 2 - 5 } & Reflexivity & 4.34 & 4.46 & not \\
\hline
\end{tabular}


\begin{tabular}{|l|l|l|l|l|}
\hline & & & significant \\
\hline
\end{tabular}

Significantly positive changes in the first group were found by applying Wilcoxon's Tcriterion.

If speaking about conceptualization style, typical changes were expressed by the value increase, whereas non-typical change (so called "zero shift") appeared significantly rarely:

$$
T_{\text {Emp }}=74, p \leq 0.01
$$

If comparing conceptualization style mean values (from 0.32 to 0.78 ), the tendency in synthetic style being changed into analytic one can be observed, i.e., subjective criteria in words estimation are changed into objective ones.

In case of reflexivity in the same way as for conceptualization style, typical changes were expressed by the value increase, whereas non-typical change (so called "zero shift") appeared significantly rarely:

$$
T_{\text {Emp }}=55, p \leq 0.01
$$

If comparing reflexivity mean values "before" and "after" project tasks fulfilling (4.32 and 5.34), the changes in the results can also be observed. Although both results are mean values, the first result is the lower bound whereas the second one is average. This fact proves the reduction of reflexivity inaccuracy.

The control group (the described tasks were not raised) is characterized by zero shift as a typical change and the value increase (appeared significantly rarely) as a non-typical change in conceptualization style:

$$
T_{\text {Emp }}=194, p \leq 0.01
$$

The conceptualization style variables $(0.34$ and 0.42$)$ indicate the lack of changes in synthetic style and subjective estimation.

As well, zero shift was a typical change and the value increase (appeared significantly rarely) was a non-typical change characteristic of reflexivity:

$$
T_{\text {Emp }}=285, p \leq 0.01
$$

In other words both results are at the lower bound of reflexivity mean value.

Thus the results of the research allow drawing the following conclusions: contemporary education of the future IT-specialists aimed at training a new type of engineers (conscious and intelligent) improves in case of using reflexive-integrative approach thanks to the tasks developing conceptual (categoric) abilities and reflexivity as being characteristic of intellectual and personal resource. Positive changes in reflexivity indices, categoric abilities, conceptual style (synthetic style changes into analytic one) as well as in the students' roles integration (author-developer, expert, researcher) are determined by using the reflexive-integrative approach in teaching the future IT-specialists including but not limited to fulfilling such task as developing the set of words describing specific concept (the "education" concept in this particular case).

It is necessary to form and to develop such functional characteristic as analytic conceptual style and to reduce reflexivity inaccuracy by applying the reflective and integral approach to the learning process. This helps the students to point out essential characteristics of the notion, find out the connections between these characteristics and to interpret them by synthesis as well as to develop conceptual categoric abilities. 


\section{References}

1. A. Zahirović Suhonjić, M. Despotović-Zrakić, A. Labus, Z. Bogdanović, D. Barać, Fostering students' participation in creating educational content through crowdsourcing. Interactive Learning Environments, 27 (1), $72-85$ (2019) DOI: 10.1080/10494820.2018.1451898

2. P. Horvath, New ideas in psychology, 51, 15-20 (2018)

3. E. Zhestkova, Environment. Technology. Resources. Rezekne. Latvia Proceedings of the $11^{\text {th }}$ International Scientific and Practical Conference, 2, 180-184 (2017) DOI: $10.17770 /$ etr2017vol2.2515

4. I.A. Kibal'chenko, Students' learning and cognitive experience integration: structure, dynamics, technologies (Rostov-on-Don, 2011)

5. S.D. P'iankova, Theory of development: Differential and integrative paradigm. Acquisition: N.I. Chuprikova. Moscow: The languages of Slav cultures, 103-115 (2009)

6. M.A. Kholodnaia, Mental resources of a person: theoretic and applied researches. Materials of the Third International Academic Conference (2016)

7. M.A. Kholodnaia, Cognitiva styles. The nature of individual mind (Piter, SaintPetersburg, 2004)

8. E.V. Volkova, Technologies of mental resources development (Institute of Psychology of Russian Academy of Sciences, Moscow, 2016)

9. A.V. Karpov, Psychological journal, 24 (5), 45-57 (2003) 